\title{
Biochemical studies on the effect of nano particles of some nutrients on apoptosis modulation of breast cancer cells in experimental animals
}

\author{
Afaf Ezzat ${ }^{1}$, Abdo Abdeelhamid ${ }^{2}$, Ahmed Amin ${ }^{2}$, Amal Abdelazim¹, Mohamed Ragai ${ }^{3}$ and Dina Mohamed ${ }^{2}$ \\ ${ }^{1}$ Nutrition and Food Sciences Department, National Research Centre, Dokki, Cairo, Egypt \\ 2 Faculty of Science, Cairo University, Egypt \\ ${ }^{3}$ Inorganic Chemistry Department, National Research Centre, Cairo, Egypt
}

\section{III}

Background: Nano micronutrients and their relationship to health or disease is one of the domains of modern needs of many of extensive researches and studies. Aim: This work was carried out to study the effect of some nano nutrients to induce apoptosis in breast cancer and the inhibitory effect of them on development of toxic material of mammary tumor for 180 days against injected intraperitoneally a single dose of $(50 \mathrm{mg} / \mathrm{kg}) \mathrm{N}$-methyl-N-nitrosourea (MNU) in female rats. Experimental animals in this study divided into two categories, first category: include (78 rats) which induced breast cancer by injecting a single dose of MNU intraperitoneally. Two weeks after MNU treatment, a time by which the animals had recovered from MNU-induced toxicity, the rats were divided into 13 groups (6 for each). Finally, all the experimental results evaluated, tabulated and statistically analyzed. Results: Parameters in normal treatments showed agreement with the results of many previous biochemical studies, especially the results of folic acid, vitamin $\mathrm{C}$ and apoptosis. While the biochemical results of nano treatments showed many unexpected differences in cancer diseases, especially increasing the level of folic acid, vitamin B12 and vitamin C. There was a high release of those nano nutrients from the diseased cells. In addition to the inability of the diseased cells to keep them, which may be in a different the status for the natural form. The treatment of nano yeast showed nearly the best results among all other treatments. We will need more research to support these results. Results of nano treatment on diseased cancer cells and normal cells indicated to difference in biochemical pathways from normal cells. This will require further studies. Conclusion: Parameters in normal treatments showed agreement with the results of many previous biochemical studies, while the biochemical results of nano treatments showed many unexpected differences in cancer diseases. The treatment of nano yeast showed nearly the best results among all other treatments.

Keywords: Apoptosis; Breast Cancer; Mammary tumor; Nano Particles; Nutrients

Editor-in-Chief: Prof. M.L. Salem, PhD - Article DOI: 10.21608/jcbr.2021.60576.1149 